\title{
Characteristics of Physical Fitness and Cardiometabolic Risk in Chinese University Students with Normal-Weight Obesity: A Cross-Sectional Study
}

This article was published in the following Dove Press journal:

Diabetes, Metabolic Syndrome and Obesity: Targets and Therapy

\begin{abstract}
Qianqian Tian,' Hui Wang, ${ }^{2}$
Keneilwe Kenny Kaudimba, (D) Shanshan Guo,' Haixin Zhang,' Song Gao,' Ruwen Wang,' Xin Luan,' Jason Kai Wei Lee, (iD ${ }^{3-5}$ Peijie Chen,' Tiemin Liu, ${ }^{2}$ Ru Wang'

'School of Kinesiology, Shanghai University of Sport, Shanghai, People's Republic of China; ${ }^{2}$ State Key Laboratory of Genetic Engineering, Department of Endocrinology and Metabolism, Institute of Metabolism and Integrative Biology, Human Phenome Institute, School of Life Sciences, Zhongshan Hospital, Fudan University, Shanghai, People's Republic of China; ${ }^{3}$ Department of Physiology, Yong Loo Lin School of Medicine, National University of Singapore, Singapore; ${ }^{4}$ Global Asia Institute, National University of Singapore, Singapore; ${ }^{5}$ N.I Institute for Health, National University of Singapore, Singapore
\end{abstract}

Correspondence: Tiemin Liu State Key Laboratory of Genetic Engineering, Department of Endocrinology and Metabolism, Institute of Metabolism and Integrative Biology, Human Phenome Institute, School of Life Sciences, Zhongshan Hospital, Fudan University, Shanghai, People's Republic of China

Tel/Fax +86-2I- 3I246765

Email tiemin_liu@fudan.edu.cn

Ru Wang

School of Kinesiology, Shanghai University of Sport, Shanghai, People's Republic of China

Tel/Fax +86-2I-6550735 I

Email wangru@sus.edu.cn
Purpose: To (i) describe the characteristics of physical fitness and cardiometabolic risk in Chinese university students with normal-weight obesity (NWO); (ii) assess the association between NWO with physical fitness and cardiometabolic risk.

Methods: A total of 249 participants from the 2018 Shanghai University of Sport Alumni Health Cohort Study in China formed the study cohort. NWO was defined as normal body mass index (17.9-23.9 kg/m ${ }^{2}$ for males; $17.2-23.9 \mathrm{~kg} / \mathrm{m}^{2}$ for females) but with excess bodyfat percentage ( $\geq 20 \%$ for males; $\geq 30 \%$ for females). Body composition was assessed by dual-energy X-ray absorptiometry. Physical-fitness scores were calculated from a battery of sex-specific tests. Cardiometabolic risk was calculated using a continuous clustered risk (MetS) score.

Results: We found that $16.7 \%$ of male and $45.3 \%$ of female university students were classified as having NWO. Participants with NWO showed worse cardiorespiratory fitness

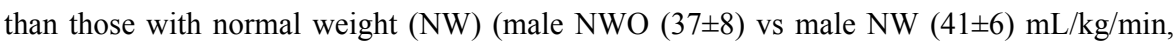
$p<0.01$; female NWO $(31 \pm 4)$ vs female NW $(33 \pm 6) \mathrm{mL} / \mathrm{kg} / \mathrm{min}, p<0.01)$. NWO was associated negatively with the fitness score $(\mathrm{OR}=0.853 ; 95 \% \mathrm{CI}=0.793-0.917)$ and was associated positively with the MetS score $(1.280 ; 1.162-1.410)$.

Conclusion: University students with NWO were characterized by higher cardiometabolic risk and lower levels of physical fitness. Our findings implied that university students with NWO should be mindful of the potential hidden health risks of excess body fat and participate in exercise to enhance physical fitness.

Keywords: body composition, body mass index, body fat distribution, skeletal muscle, public health

\section{Introduction}

Obesity is a major risk factor for non-communicable diseases worldwide, including cardiovascular disease, type-2 diabetes mellitus, and certain types of cancer. ${ }^{1}$ Weight gain from early-to-middle adulthood is associated with an increased risk of major chronic diseases and mortality, ${ }^{2,3}$ which highlights the importance of maintaining a healthy weight throughout life beginning from early adulthood.

Body mass index (BMI) is a relatively simple, low-cost, indirect measurement of obesity. Nevertheless, it cannot distinguish between lean mass (LM) and fat mass (FM) and it does not provide an indication of the distribution of body fat (BF). ${ }^{4}$ 
Dual-energy X-ray absorptiometry (DXA) can be used to evaluate body composition for FM and LM. ${ }^{5}$ An excess of fat is associated with insulin resistance, altered metabolism of lipids, and endothelial dysfunction. ${ }^{6}$ Individuals with normal body weight according to BMI but having a high percentage of BF are said to have "normal-weight obesity" (NWO). Compared with their normal weight (NW) lean counterparts, individuals with NWO carry a higher risk of developing metabolic syndrome, cardiometabolic dysfunction, and death. ${ }^{7}$

Increased $\mathrm{FM}$ and body fat percentage $(\mathrm{BF} \%)$ are associated with cardiometabolic risk, and are also the primary markers of worse physical performance. ${ }^{8}$ Children and adolescents suffering from obesity have lower cardiorespiratory fitness and lower body explosive strength. ${ }^{9}$ Higher LM (which corresponds to muscle mass) is related to higher levels of physical fitness. ${ }^{10}$ Conversely, individuals with low cardiorespiratory fitness exhibit an increased risk of low LM. ${ }^{11}$ Physical fitness is, therefore, an important marker of health. A lower level of physical fitness early in life is a key risk factor for long-term cardiovascular disease and death. ${ }^{12}$ NWO is a risk factor for cardiovascular disease, metabolic dysregulation, and poor functional outcomes, but the relationship between physical fitness and NWO in young people is not known.

We wished to (i) describe the characteristics of physical fitness and cardiometabolic risk among Chinese university students with NWO; (ii) assess the association between NWO with physical fitness and cardiometabolic risk.

\section{Materials and Methods Study Design}

This cross-sectional study was part of the 2018 Shanghai University of Sport (SUS) Alumni Health Cohort Study in Shanghai, China. The 2018 SUS Alumni Health Cohort Study is a prospective longitudinal, observational cohort study to assess changing patterns of physical exercise, body weight, and physical fitness from early-to-middle adulthood and their relationship to major chronic diseases in the Chinese population. It comprised athletic students and non-athletic students enrolled during Fall 2018 from SUS.

All participants provided written informed consent. The study was conducted in accordance with the ethical principles stated in the Declaration of Helsinki 1964 and its later amendments. The Ethics Review Board at SUS approved the study protocol (2018047). In addition, the study was registered at the Chinese Clinical Trial Register (ID: ChiCTR2000028981).

\section{Determination of Sample Size}

The formula for estimating the sample size is shown below. Expecting a 95\% confidence interval (CI), we used a prevalence of NWO of $28.2 \%$ based on a study by Romero-Corral and coworkers. ${ }^{13}$ The required sample size was $\geq 245$ :

$$
\begin{aligned}
n & =Z \alpha / 2^{2} * P(1-P) / \delta^{2} \\
& =1.96^{2} * 0.282 *(1-0.282) / 0.0564^{2} \approx 245
\end{aligned}
$$

where $\alpha=0.05$, confidence level $=1-\alpha=95 \%, Z \alpha / 2=$ $1.96, \mathrm{P}$ is the prevalence of $\mathrm{NWO}=0.282$, and $\delta$ is the permissible error $=0.2 \mathrm{P}=0.0564$.

\section{Participants}

A total number of 909 first-year university students were included in the 2018 SUS Alumni Health Cohort Study. Student athletes $(n=496)$ were excluded because their routine training would affect the outcome measures. Out of 413 university students, 382 participants had data for BMI and $\mathrm{BF} \%$ that allowed their classification. The other 31 participants were excluded from the analysis because data on BMI or BF\% were not available. The remaining 382 first-year university students were divided into four groups according to definitions of NW and NWO: male NW ( $\mathrm{n}=$ 56), male NWO $(\mathrm{n}=21)$, female NW $(\mathrm{n}=83)$ and female NWO $(n=116)$. Twenty-seven participants were excluded because data on physical fitness or blood samples were missing. Finally, 249 participants were included in the data analysis: male NW $(\mathrm{n}=53)$, male NWO $(\mathrm{n}=20)$, female NW $(n=73)$ and female NWO $(n=103)$ (Figure 1). The normal cutoff point of BMI (in $\mathrm{kg} / \mathrm{m}^{2}$ ) was $17.9 \leq \mathrm{BMI} \leq$ 23.9 for men and $17.2 \leq \mathrm{BMI} \leq 23.9$ for women based on National Students Constitutional Health Standards (2014 revised edition) in China. ${ }^{14}$ The cutoff for obesity using BF\% was $\geq 20 \%$ for males and $\geq 30 \%$ for females based on definitions set by the World Health Organization. ${ }^{15}$

\section{Anthropometry and Body Composition}

The weight (precision, $0.1 \mathrm{~kg}$ ) and height (precision, $0.1 \mathrm{~cm}$ ) of participants were measured in light clothing without shoes or socks. LM and FM of the whole body, android region, and gynoid region were assessed using DXA using a Prodigy ${ }^{\mathrm{TM}}$ Lunar system (General Electric, Boston, MA, USA). DXA was done from head to toe with 
The 2018 SUS Alumni Health Cohort Study in Shanghai, China

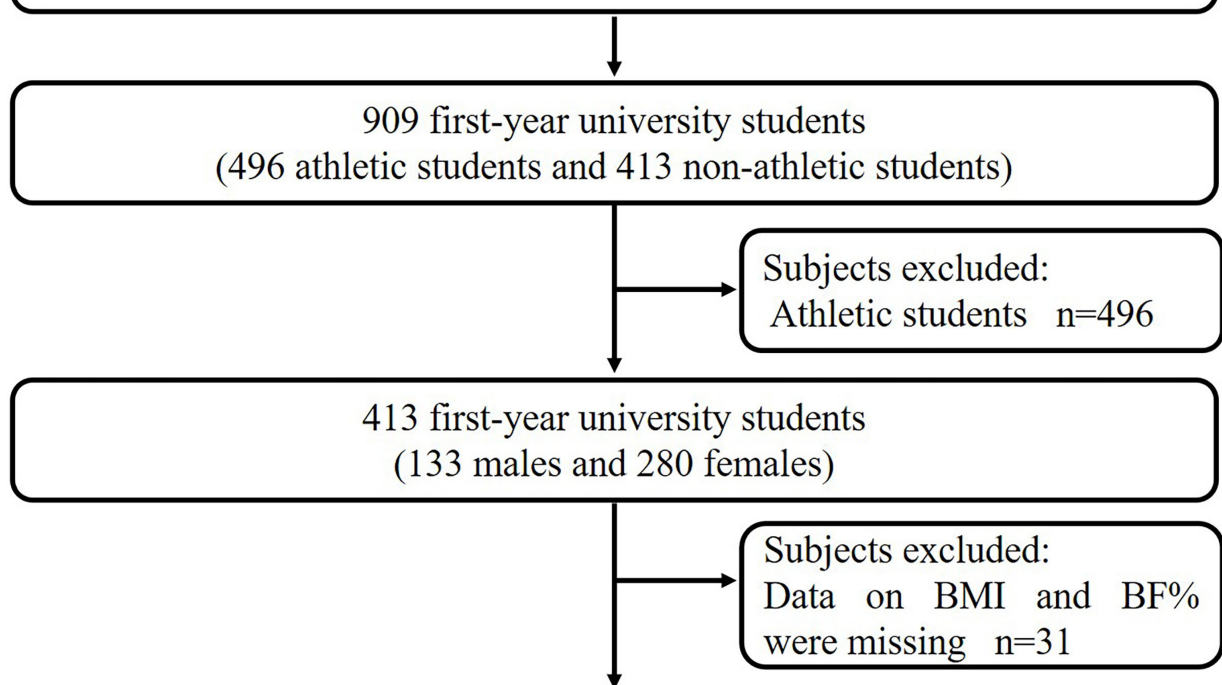

382 first-year university students

(126 males and 256 females)

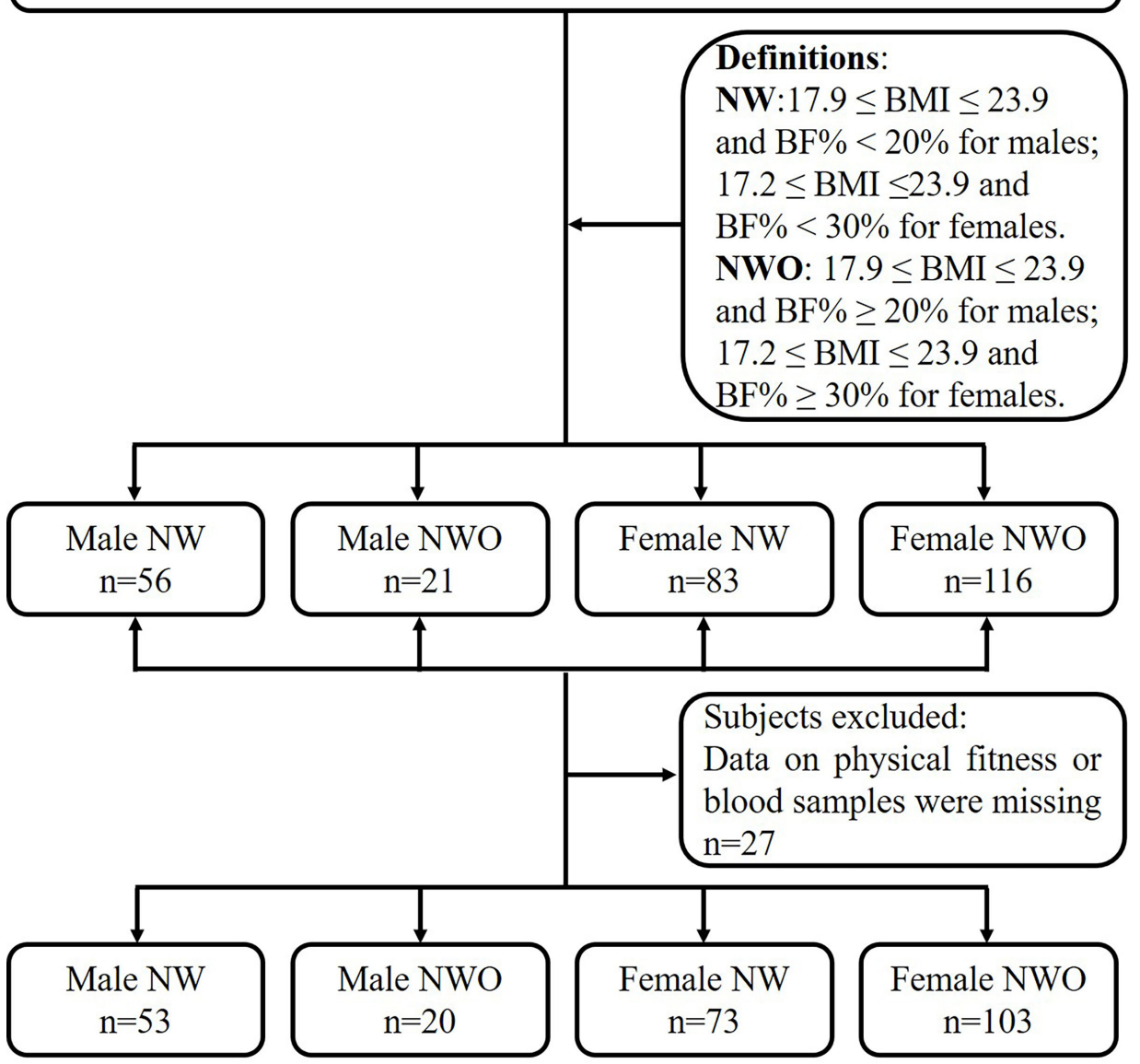

Figure I Flowchart of our study.

Abbreviations: BF\%, body fat percentage; BMI, body mass index; NW, normal weight; NWO, normal-weight obesity; SUS, Shanghai University of Sport. 
participants supine and arms by their side. Variables of interest from DXA were LM and FM of the whole body, android region, and gynoid region.

\section{Blood Pressure}

Participants were required to sit with their feet resting flat on the ground for $5 \mathrm{~min}$ before the first blood-pressure measurement. Two measurements were taken $\geq 1$-min apart using an electronic sphygmomanometer (UDEX-Twin Type 2; Japan). The mean value of the measurements was used for analyses. If the two measurements differed by $\geq 5 \mathrm{mmHg}$, an additional measurement was taken with the mean between the two closest readings used for the analysis. ${ }^{16}$ Mean arterial pressure (MAP) was estimated from the formula:

MAP $=([(2 \times$ diastolic blood pressure $)+$ systolic blood pressure $] / 3$ )

\section{Physical Fitness}

The physical fitness of participants was assessed using a Physical-Fitness Test System (Takei Kiki Kogyo, Tokyo, Japan). Before testing of each item of physical fitness, participants were asked to rest for $5 \mathrm{~min}$.

The physical-fitness test comprised several test items. Upper-limb muscle strength was evaluated by a handgrip dynamometer (accuracy $=0.1 \mathrm{~kg}$ ). Two attempts were made on each hand, with the higher of the two scores used for analyses. Respiratory function was assessed by a portable dynamic spirometer (accuracy $=1 \mathrm{~mL}$ ). The "whole-body reaction time" (WBRT) was the time required from visual stimuli to when the participant lifted his/her leg from a starting platform. The WBRT was evaluated by a reaction-time instrument (reading accuracy $=0.001 \mathrm{~s}$ ). Hamstring flexibility was assessed by a sit-andreach test. In a seated position with both knees extended fully and feet placed firmly against a vertical support, participants were asked to reach forward with their hands. Two attempts were made, and the highest two scores used for analyses (measured to the nearest $0.1 \mathrm{~cm}$ ). Static balance was evaluated by standing on one leg for as long as possible with eyes closed; the accuracy of the reading was $1 \mathrm{~s}$, and the longer of the two attempts was recorded. Cardiorespiratory endurance was assessed by maximal oxygen consumption $\left(\mathrm{VO}_{2 \max }\right)$, and the accuracy of the reading was $1 \mathrm{~mL} / \mathrm{kg} / \mathrm{min}$. A graded cycleergometry test was undertaken for $13 \mathrm{~min}$ at increasing intensity based on a submaximal heart rate. During this time, the heartbeat was monitored, and built-in software calculated the $\mathrm{VO}_{2 \max }$. Abdominal endurance was evaluated by 1-min sit-ups. Explosive power was assessed by a standing long jump. The participants had two trials, and the better score was used for statistical analyses.

The fitness score was the sum of the sex-specific z-score for the test items of physical fitness stated above. A higher fitness score indicated a better level of physical fitness.

\section{Blood Analyses}

After an overnight fast of $\geq 8 \mathrm{~h}, 10 \mathrm{~mL}$ of venous blood was drawn from participants and analyzed at Shanghai Yulong Medical Laboratory (Shanghai, China). The concentration of fasting blood glucose (FBG) and concentration of lipids were measured through standard methods of enzymatic colorimetry. All assay kits were obtained from Qufu Yulong Biotechnology (Shandong, China) and levels of fasting blood cholesterol (CHOL), triglycerides (TG), high-density lipoprotein-cholesterol (HDL-C) and lowdensity lipoprotein-cholesterol (LDL-C) were measured.

\section{Assessment of Cardiometabolic Risk}

To assess cardiometabolic risk, we constructed a standardized, continuously distributed variable for the clustered metabolic risk (MetS) score. ${ }^{17}$ The latter was the sum of the $z$ scores for MAP, android FM, FBG, TG, CHOL, HDL-C* -1 and LDL-C. A higher MetS score indicates a worse cardiometabolic profile.

\section{Statistical Analyses}

Participant characteristics, physical fitness, body composition, glucose level, and lipid variables are reported as the mean \pm standard deviation and 95\% CI. Characteristic differences in NW and NWO within the same sex were tested by the Mann-Whitney non-parametric test. Binary logistic regression analysis was undertaken to examine the associations between NWO, physical fitness, and cardiometabolic risk. The Spearman correlation coefficient between body composition, cardiometabolic risk factors, and physical fitness was calculated. Analyses were performed in IBM SPSS Statistics 22.0 software (IBM SPSS Inc., Chicago, Illinois). $p<0.05$ indicated significance.

\section{Results}

\section{Basic Characteristics of Participants}

The study cohort was 382 (126 males and 256 females) first-year university students in the 2018 SUS Alumni 
Health Cohort Study. Two (1.6\%) males and nine (3.5\%) females were classified as underweight, $77(61.1 \%)$ and $199(77.7 \%)$ as NW, 31 (24.6\%) and 35 (13.7\%) as overweight, and $16(12.7 \%)$ and $13(5.1 \%)$ as obese, respectively. We focused on participants with NW according to BMI and participants with NW according to BMI but with a high $\mathrm{BF} \%$. Of the participants who were classified as NW by BMI, and according to the cutoff point of BF\%, 56 (44.4\%) males and 83 (32.4\%) females were classified as NW, and $21(16.7 \%)$ males and $116(45.3 \%)$ females were classified as NWO.

The characteristics of participants classified as NW and NWO are shown in Table 1. Age, height, systolic blood pressure, diastolic blood pressure, and MAP between NW and NWO were similar in males and females. Compared with males of NW, males of NWO had a higher body mass $(p<0.05)$. Females with NWO had a higher body mass than females with NW $(p<0.01)$. Males and females with NWO had higher $\mathrm{BMI}$ and $\mathrm{BF} \%$ than males and females with NW $(p<0.01)$.

\section{Physical Fitness Between People with NW and People with NWO}

The characteristics of physical fitness in people with NW and people with NWO are shown in Table 2. Males with NWO showed worse performance for sitting and reaching than individuals with NW $(p<0.05)$. Differences were not observed between females with NWO and females with NW for sitting and reaching. Males with NWO had lower $\mathrm{VO}_{2 \max }$ than males with $\mathrm{NW}(p<0.01) . \mathrm{VO}_{2 \max }$ was similar among females $(p<0.01)$. Females with NWO carried out fewer 1-min sit-ups than females with NW $(p<0.05)$. In terms of the standing long jump, males and females with NWO showed worse performance than that of males and females with NW ( $p<0.01$ for all). Differences were not observed between males and females with NWO and males and females with NW for handgrip strength, forced vital capacity, WBRT, or standing on one leg with eyes closed. Males and females with NWO had a lower fitness score than males and females with NW ( $p<0.01$ for all) (Figure 2B).

\section{Body Composition Between People with NW and People with NWO}

Differences in total and regional LM and FM are shown in Table 2. FM in the gynoid (lower body) region and android region (abdominal area) of males and females with NWO were higher compared with males and females with NW ( $p<0.01$ for all). Males and females with NWO had greater total FM than males and females with NW $(p<0.01$ for all). Total LM of males with NWO was lower than that of males with NW $(p<0.05)$. Total LM was similar in females with NWO and NW. Compared with the NW group, the LM/FM ratio was lower in the NWO group ( $p<0.01$ for all).

\section{Glucose Level, Levels of Lipids, and Cardiometabolic Risk}

The different levels of glucose and lipids are displayed in Figure 2A. Levels of LDL-C in females with NWO were higher than those of females with NW $(p<0.05)$, whereas LDL-C levels were similar in males with NWO and in males with NW. Levels of FBG, CHOL, TG, and HDL-C were similar between the NWO group and NW group in males and females. However, males and females with

Table I Basic Characteristics of Study Participants

\begin{tabular}{|c|c|c|c|c|}
\hline \multirow{2}{*}{ Characteristics } & \multicolumn{2}{|l|}{ Male $(n=73)$} & \multicolumn{2}{|l|}{ Female $(n=177)$} \\
\hline & $N W(n=53)$ & NWO $(n=20)$ & $N W(n=73)$ & NWO (n = 103) \\
\hline Age (years) & $18.5 \pm 0.7(18.3,18.7)$ & $18.6 \pm 0.8(18.2,18.9)$ & $18.2 \pm 0.7(18.1,18.4)$ & $18.2 \pm 0.7(18.1,18.4)$ \\
\hline Height $(\mathrm{cm})$ & $175 \pm 7(173,177)$ & $176 \pm 5(173,178)$ & $162 \pm 5(161,164)$ & $162 \pm 6(161,164)$ \\
\hline Body weight (kg) & $63.8 \pm 6.4(62.1,65.6)$ & $67.9 \pm 4.8^{*}(65.7,70.1)$ & $51.3 \pm 4.3(50.3,52.3)$ & $55.4 \pm 6.0 * *(54.3,56.6)$ \\
\hline BMI $\left(\mathrm{kg} / \mathrm{m}^{2}\right)$ & $20.8 \pm 1.5(20.4,21.3)$ & $22.0 \pm 1.4^{* *}(21.4,22.7)$ & $19.4 \pm 1.0(19.2,19.7)$ & $21.0 \pm 1.6 * *(20.7,21.3)$ \\
\hline $\mathrm{BF} \%$ & $13 \% \pm 3 \%(12 \%, 14 \%)$ & $23 \% \pm 3 \% * *(22 \%, 24 \%)$ & $27 \% \pm 2 \%(26 \%, 27 \%)$ & $34 \% \pm 3 \% * *(33 \%, 34 \%)$ \\
\hline $\mathrm{SBP}(\mathrm{mmHg})$ & $127 \pm 14(123,131)$ & $121 \pm 14(115,128)$ & $109 \pm 10(106,111)$ & $111 \pm 13(109,114)$ \\
\hline $\mathrm{DBP}(\mathrm{mmHg})$ & $76 \pm 13(73,80)$ & $81 \pm 14(75,88)$ & $68 \pm 9(66,7 I)$ & $70 \pm 11(68,72)$ \\
\hline MAP $(\mathrm{mmHg})$ & $93 \pm 11(90,96)$ & $95 \pm 13(89,100)$ & $82 \pm 9(80,84)$ & $84 \pm 10(82,86)$ \\
\hline
\end{tabular}

Notes: Data are the mean \pm SD and $95 \% \mathrm{Cl}$. ${ }^{*} p<0.05$, ${ }^{* *} p<0.01$ compared with NW of the same sex (male NW vs male NWO, female NW vs female NWO) Abbreviations: BF\%, body fat percentage; BMI, body mass index; DBP, diastolic blood pressure; MAP, mean arterial pressure; NW, normal weight; NWO, normal-weight obesity; SBP, systolic blood pressure. 
Table 2 Characteristics of Physical Fitness and Body Composition in People with NW and People with NWO

\begin{tabular}{|c|c|c|c|c|}
\hline \multirow[t]{2}{*}{ Characteristics } & \multicolumn{2}{|l|}{ Male $(n=73)$} & \multicolumn{2}{|l|}{ Female $(n=177)$} \\
\hline & $N W(n=53)$ & NWO $(n=20)$ & $N W(n=73)$ & NWO $(n=103)$ \\
\hline \multicolumn{5}{|l|}{ Physical fitness } \\
\hline Handgrip strength (kg) & $38.9 \pm 5.5(37.4,40.4)$ & $37.6 \pm 3.2(36.1,39.1)$ & $24.4 \pm 4.2(23.5,25.4)$ & $23.4 \pm 3.9(22.7,24.2)$ \\
\hline Forced vital capacity $(\mathrm{mL})$ & $3187 \pm 833(2957,3417)$ & $3222 \pm 927(2788,3656)$ & $2314 \pm 516(2194,2435)$ & $2413 \pm 558(2304,2522)$ \\
\hline Whole-body reaction time (s) & $0.392 \pm 0.055(0.376,0.407)$ & $0.405 \pm 0.046(0.383,0.426)$ & $0.406 \pm 0.062(0.392,0.421)$ & $0.42 I \pm 0.052(0.4 \mid I, 0.43 I)$ \\
\hline Sit and reach $(\mathrm{cm})$ & $14.7 \pm 6.9(12.8,16.6)$ & $11.3 \pm 5.8 *(8.5,14.0)$ & $16.0 \pm 6.4(14.5,17.5)$ & $14.9 \pm 6.9(13.6,16.3)$ \\
\hline $\begin{array}{l}\text { Standing on one leg with } \\
\text { eyes closed (s) }\end{array}$ & $70 \pm 57(54,86)$ & $59 \pm 67(28,90)$ & $63 \pm 56(50,76)$ & $56 \pm 57(45,67)$ \\
\hline $\mathrm{VO}_{2 \max }(\mathrm{mL} / \mathrm{kg} / \mathrm{min})$ & $41 \pm 6(40,43)$ & $37 \pm 8^{* *}(33,40)$ & $33 \pm 6(32,35)$ & $31 \pm 4^{* *}(30,32)$ \\
\hline Sit-ups (reps/min) & $40 \pm 9(38,43)$ & $38 \pm 10(33,42)$ & $33 \pm 11(31,36)$ & $29 \pm 9^{*}(28,31)$ \\
\hline Standing long jump (cm) & $235 \pm 19(230,240)$ & $217 \pm 16 * *(209,224)$ & $174 \pm 18(169,178)$ & $162 \pm 16 * *(159,165)$ \\
\hline \multicolumn{5}{|l|}{ Body composition } \\
\hline Android FM (kg) & $0.7 \pm 0.2(0.7,0.8)$ & $1.5 \pm 0.2 * *(1.4,1.6)$ & $1.0 \pm 0.2(0.9,1.0)$ & $1.4 \pm 0.3^{* *}(1.3,1.4)$ \\
\hline Gynoid FM (kg) & $1.7 \pm 0.5(1.6,1.9)$ & $2.8 \pm 0.4 * *(2.6,3.0)$ & $3.0 \pm 0.4(2.9,3.1)$ & $3.7 \pm 0.6 * *(3.6,3.8)$ \\
\hline Total FM (kg) & $8.1 \pm 2.4(7.4,8.7)$ & $14.9 \pm 2.4^{* *}(13.8,16.0)$ & $13.1 \pm 1.9(12.7,13.5)$ & $17.7 \pm 2.8^{* *}(17.1,18.2)$ \\
\hline Total LM (kg) & $52.7 \pm 5.3(51.2,54.1)$ & $49.5 \pm 3.4^{*}(47.9,51.1)$ & $35.4 \pm 2.9(34.8,36.1)$ & $34.8 \pm 3.6(34.1,35.5)$ \\
\hline LM/FM ratio & $7.1 \pm 2.3(6.5,7.8)$ & $3.4 \pm 0.5^{* *}(3.2,3.6)$ & $2.7 \pm 0.4(2.7,2.8)$ & $2.0 \pm 0.2^{* *}(2.0,2.0)$ \\
\hline
\end{tabular}

Notes: Data are the mean \pm SD and $95 \% \mathrm{Cl} .{ }^{*} p<0.05,{ }^{* *} p<0.01$ compared with NW of the same sex (male NW vs male NWO, female NW vs female NWO)

Abbreviations: FM, fat mass; LM, lean mass; NW, normal weight; NWO, normal-weight obesity; $\mathrm{VO}_{2 \text { max }}$, maximal oxygen consumption.

NWO had a greater cardiometabolic risk than males and females with NW ( $p<0.01$ for all) (Figure 2B).

\section{Associations of NWO with Physical}

\section{Fitness and Cardiometabolic Risk}

The relationship between NWO with physical fitness and cardiometabolic risk is shown in Table 3. NWO was associated negatively with the fitness score $(p=0.000)$. NWO was associated positively with the MetS score $(p=0.000)$. For every 1-unit increase in the MetS score, the risk of NWO prevalence increased by $28.0 \%$.

\section{Correlations Between Body Composition, Cardiometabolic Risk Factors, and Physical Fitness}

The correlations between FM, LM, cardiometabolic risk factors, and physical fitness are displayed in Figure 3. Android FM, gynoid FM, and total FM were correlated positively with cardiometabolic risk factors such as the glucose level, levels of lipids, and MetS score. Total LM and the LM/FM ratio were correlated negatively with cardiometabolic risk factors. With respect to physicalfitness items, handgrip strength, forced vital capacity, standing on one leg with eyes closed, $\mathrm{VO}_{2 \max }$, sit-ups, standing long jump, and fitness score were correlated negatively with FM (android FM, gynoid FM, total FM) and correlated positively with LM (total LM and LM/FM ratio).

\section{Discussion}

There is limited information on the physical fitness and biological characteristics of Chinese young people with NWO. We assessed the levels of physical fitness and cardiovascular risk factors in university students with NW or NWO.

The key finding of our study was that, out of 382 university students evaluated, $16.7 \%$ males and $45.3 \%$ females were classified as having NWO. In addition, university students with NWO were characterized by lower levels of physical fitness and greater cardiovascular risk compared with their NW counterparts.

Using a cutoff point (highest tertile) for classification of NWO, the prevalence was $33.3 \%$ for women and $23.1 \%$ for men in Americans aged $\geq 20$ years in a study by Romero-Corral and colleagues. ${ }^{13}$ In Swiss adults aged $35-75$ years, NWO prevalence varied from $1.4 \%$ to $27.8 \%$ in women according to the cutoff point used, but the NWO prevalence was $<1 \%$ in men for all thresholds used. ${ }^{18}$ However, a national study in Chinese individuals aged $\geq 20$ years found that NWO prevalence in different age groups ranged from $6.7 \%$ to $10.2 \%$ in males and $1.7 \%$ to $6.6 \%$ in females using various definitions. ${ }^{19}$ The 

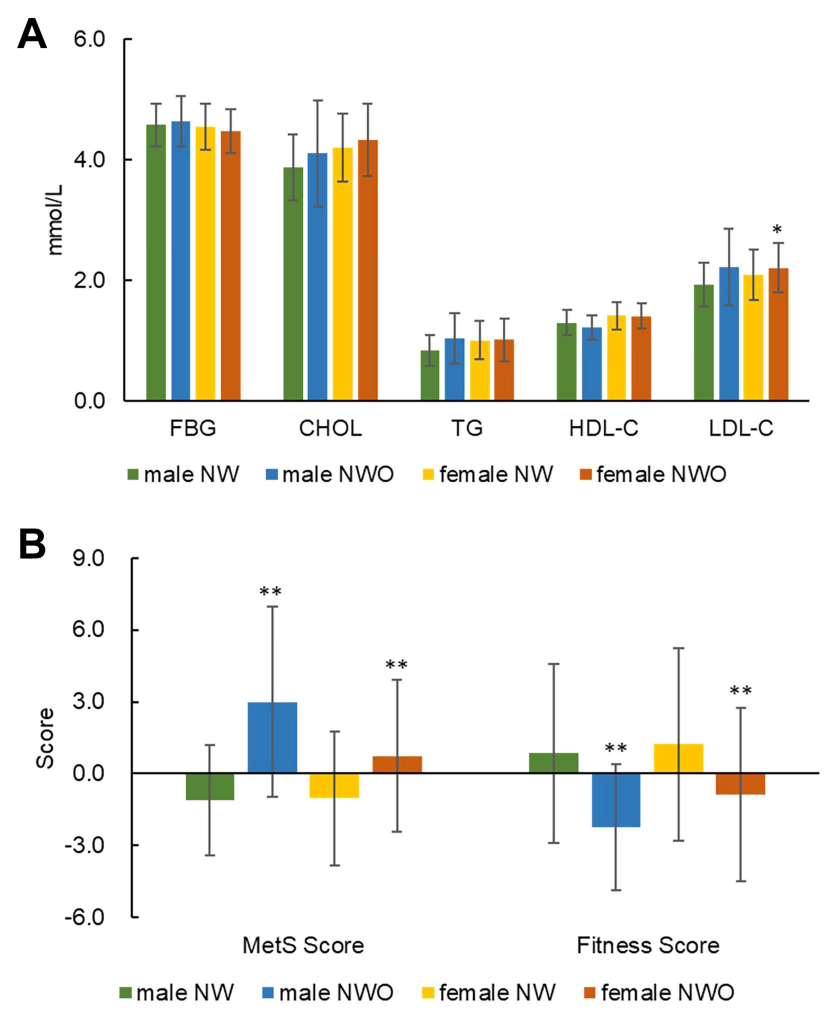

Figure 2 Glucose level and levels of lipids (A), fitness score and MetS score (B) in NW and NWO.

Notes: Data are the mean $\pm S D$. $*_{p}<0.05, * * p<0.01$ compared with NW of the same sex (male NW vs male NWO, female NW vs female NWO). The fitness score was the sum of the sex-specific z-score for physical-fitness test items (handgrip strength, forced vital capacity, whole-body reaction time, sit and reach, standing on one leg with eyes closed, maximal oxygen consumption, sit-ups, and standing long jump). A higher fitness score indicates a better level of physical fitness. The MetS score was the sum of the z-score of the following metabolic traits: mean arterial pressure $([(2 \times$ diastolic blood pressure $)+$ systolic blood pressure $] / 3)$; android FM; FBG; TG, CHOL, HDL-C*-I and LDL-C. A higher MetS score indicates a worse cardiometabolic profile.

Abbreviations: $\mathrm{CHOL}$, cholesterol; FBG, fasting blood glucose; HDL-C, highdensity lipoprotein-cholesterol; LDL-C, low-density lipoprotein-cholesterol; NW, normal weight; NWO, normal-weight obesity; TG, triglycerides.

differences among these studies were due to the different definitions used, so their results cannot be compared directly. In addition, data for NWO prevalence differ between studies because of ethnicity, age, sex differences in populations, and the lack of consensus on diagnostic criteria. Our study indicated that NWO prevalence in female university students was higher than that in male university students. Nearly half of the female university students were diagnosed as having NWO obesity, which implied an important health threat among young females.

University students with NWO were characterized by lower levels of physical fitness in our study. Physical fitness is operationalized as measurable health- and skillrelated attributes that includes cardiorespiratory fitness, muscle strength, aerobic endurance, flexibility, balance,
Table 3 Associations of NWO with Physical Fitness and Cardiometabolic Risk

\begin{tabular}{|l|l|l|l|}
\hline Outcome & OR & $\mathbf{9 5 \%} \mathbf{~ C l}$ & $\boldsymbol{P}$ \\
\hline Fitness score & 0.853 & $(0.793,0.917)$ & 0.000 \\
MetS score & 1.280 & $(1.162,1.410)$ & 0.000 \\
\hline
\end{tabular}

Notes: The fitness score was the sum of the sex-specific z-score for physicalfitness test items (handgrip strength, forced vital capacity, whole-body reaction time, sit and reach, standing on one leg with eyes closed, maximal oxygen consumption, sit-ups, and standing long jump). A higher fitness score indicates a better level of physical fitness. The MetS score was the sum of the z-score of the following metabolic traits: mean arterial pressure $([(2 \times$ diastolic blood pressure $)+$ systolic blood pressure]/3); android FM; FBG; TG, CHOL, HDL-C*-I and LDL-C. A higher MetS score indicates a worse cardiometabolic profile.

Abbreviations: $\mathrm{CHOL}$, cholesterol; $\mathrm{Cl}$, confidence interval; $\mathrm{FBG}$, fasting blood glucose; HDL-C, high-density lipoprotein-cholesterol; LDL-C, low-density lipoprotein-cholesterol; NW, normal weight; NWO, normal-weight obesity; OR, odds ratio; TG, triglycerides.

agility, reaction time, and power. ${ }^{20}$ There was a strong inverse relationship between excess body weight and physical fitness. A study carried out in Finland found that being overweight was associated negatively with aerobic endurance and muscle endurance, as well as explosive power in people aged $15-16$ years. $^{21}$ A national survey from China covering the period 1985-2014 indicated that obesity is a major driver of decline in the physical fitness of Chinese children and adolescents, including reductions in respiratory function, strength, explosive power, and cardiorespiratory endurance. ${ }^{22}$ Consistent with data from other studies, university students with NWO in our study had poor cardiorespiratory endurance and explosive power compared with their NW counterparts. Physical-fitness scores were calculated from a battery of sex-specific tests, and indicated that males and females with NWO had lower fitness scores than males and females with NW. In addition, a growing prevalence of NWO in military recruits accompanied by lower fitness is a growing concern. Fitness levels of these students can affect health and therefore operational readiness, ${ }^{23}$ because a portion of this population would be future military recruits and therefore could influence the welfare of the nation. Priority should therefore be made at poor fitness in university students and young adults with NWO.

We found that young people with NWO had higher FM and lower LM compared with those with NW. Excess FM is related to insulin resistance, dyslipidemia, and increased cardiovascular risk. ${ }^{24,25}$ However, the potential role of muscle in obesity prevention is less appreciated. ${ }^{26} \mathrm{LM}$ plays a major part in the maintenance of physical activity and fitness. Lower muscular fitness is associated with increased cardiovascular risk and metabolic risk. ${ }^{27}$ The 


\begin{tabular}{|c|c|c|c|c|c|c|}
\hline & Android FM (kg) & Gynoid FM (kg) & Total FM (kg) & Total LM (kg) & LM/FM ratio & \\
\hline $\mathrm{SBP}(\mathrm{mmHg})$ & -0.100 & $-0.219^{* *}$ & $-0.162^{*}$ & $0.497 * *$ & 0.306 ** & \\
\hline $\mathrm{DBP}(\mathrm{mmHg})$ & -0.007 & $-0.129^{*}$ & -0.072 & $0.296^{* *}$ & $0.181^{* *}$ & \\
\hline $\operatorname{MAP}(\mathrm{mmHg})$ & -0.048 & $-0.186^{* *}$ & -0.122 & $0.432^{\star \star}$ & $0.263^{* *}$ & \\
\hline $\mathrm{CHOL}(\mathrm{mmol} / \mathrm{L})$ & $0.146^{*}$ & $0.224^{* *}$ & $0.210^{\star *}$ & $-0.271^{\star *}$ & $-0.276^{* \star}$ & 1 \\
\hline $\mathrm{TG}(\mathrm{mmol} / \mathrm{L})$ & 0.094 & 0.054 & 0.076 & $-0.236^{\star *}$ & $-0.136^{*}$ & \\
\hline HDL-C (mmol/L) & 0.019 & $0.213^{* *}$ & $0.150^{*}$ & $-0.207^{* *}$ & $-0.211^{\star *}$ & 0.5 \\
\hline LDL-C (mmol/L) & $0.159^{*}$ & $0.150^{*}$ & $0.162^{*}$ & $-0.208^{\star *}$ & $-0.212^{\star *}$ & \\
\hline $\mathrm{FBG}(\mathrm{mmol} / \mathrm{L})$ & -0.011 & -0.067 & -0.054 & 0.093 & 0.104 & 0 \\
\hline MetS Score & $0.405^{* *}$ & $0.217^{* *}$ & $0.306^{* *}$ & -0.017 & $-0.235^{* *}$ & 0 \\
\hline Handgrip strength $(\mathbf{k g})$ & $-0.243^{\star *}$ & $-0.468^{\star *}$ & $-0.394^{* *}$ & $0.830 * *$ & $0.635^{* *}$ & \\
\hline Forced vital capacity (ml) & -0.076 & $-0.207^{* *}$ & $-0.160^{*}$ & $0.505^{* *}$ & $0.326^{* *}$ & -0.5 \\
\hline Whole-body reaction time (s) & 0.087 & $0.173^{* *}$ & $0.155^{*}$ & $-0.127^{*}$ & $-0.179^{* *}$ & \\
\hline Sit and reach $(\mathrm{cm})$ & 0.018 & 0.123 & 0.077 & 0.067 & -0.039 & -1 \\
\hline Standing on one leg with eyes closed (s) & $-0.140^{*}$ & -0.114 & $-0.150^{*}$ & 0.070 & 0.132 * & $\begin{array}{l}\text { Spearman } \\
\text { Correlation }\end{array}$ \\
\hline $\mathrm{VO}_{2 \max }(\mathrm{ml} / \mathrm{kg} / \mathrm{min})$ & $-0.457^{* *}$ & $-0.623^{* *}$ & $-0.597^{* *}$ & $0.373^{* *}$ & 0.606 ** & \\
\hline Sit-ups (rep/min) & $-0.283^{* *}$ & $-0.378^{* *}$ & $-0.374^{* *}$ & $0.360^{* *}$ & $0.428^{* *}$ & \\
\hline Standing long jump (cm) & $-0.410^{\star *}$ & $-0.613^{* *}$ & $-0.582^{* *}$ & $0.739^{\star *}$ & $0.750^{\star *}$ & \\
\hline Fitness Score & $-0.196^{* *}$ & -0.107 & $-0.168^{* *}$ & $0.204^{* *}$ & 0.202 ** & \\
\hline
\end{tabular}

Figure 3 Correlations between body composition, cardiometabolic risk factors, and physical fitness.

Notes: Results are Spearman correlation coefficients. ${ }^{*} p<0.05$, ${ }^{* *} p<0.01$. The fitness score was the sum of the sex-specific $z$-score for physical-fitness test items (handgrip strength, forced vital capacity, whole-body reaction time, sit and reach, standing on one leg with eyes closed, maximal oxygen consumption, sit-ups, and standing long jump). A higher fitness score indicates a better level of physical fitness. The MetS score was the sum of the z-score of the following metabolic traits: mean arterial pressure ([(2x diastolic blood pressure) + systolic blood pressure]/3); android FM; FBG; TG, CHOL, HDL-C*-I and LDL-C. A higher MetS score indicates a worse cardiometabolic profile. Abbreviations: $\mathrm{CHOL}$, cholesterol; DBP, diastolic blood pressure; FBG, fasting blood glucose; HDL-C, high-density lipoprotein-cholesterol; LDL-C, low-density lipoprotein-cholesterol; MAP, mean arterial pressure; SBP, systolic blood pressure; TG, triglycerides; $\mathrm{VO}_{2 \text { max }}$ maximal oxygen consumption.

increased cardiometabolic risk in NWO is attributable to reduced $\mathrm{LM}^{28}$ NWO was associated with poor physical fitness and the relationship was mediated (at least in part) by lower LM in Chinese university students. ${ }^{29}$ In our study, reduced LM was found in students with NWO. Total LM of males with NWO was lower than that of males with NW. Moreover, the LM/FM ratio was lower in the NWO group than that in the NW group in male and female students. Exercise is an effective strategy for treating various diseases and improving fitness, ${ }^{30-32}$ so appealing urgently to these high-risk individuals to enhance their physical fitness is important. Worldwide recommendations for physical activity call for adults aged $\geq 18$ years to complete $\geq 150$ min of moderate-intensity physical activity or $75 \mathrm{~min}$ of vigorous-intensity physical activity every week to increase fitness levels. ${ }^{33}$

An analysis of classic cardiovascular risks factors showed similar profiles between university students with NWO or NW for the glucose level, levels of lipids, and blood pressure. Nevertheless, several recent studies have reported that metabolic dysregulation increases cardiovascular risks in people classified with NWO. Women aged 35-75 years with NWO were shown to have a higher prevalence of dyslipidemia and hyperglycemia than those lean women. ${ }^{34}$ People aged $18-30$ years classified as having NWO carry an increased cardiovascular risk, including high blood pressure and low levels of HDL-C. ${ }^{35}$ In accordance with data from other studies, university students with NWO had a higher cardiometabolic risk than their NW counterparts. However, there were no differences in cardiovascular risk factors as continuous variables between NWO and NW in our study. This observation could be due to the younger age of our participants. The parameters of cardiometabolic risk in early life are associated with an increased risk of cardiometabolic morbidity and mortality in adulthood, ${ }^{15}$ so young adults should seek to preserve their cardiometabolic health. 
The relationship between NWO and metabolic risk has been characterized in middle-aged adults (low-grade proinflammatory status, increased oxidative stress, insulin resistance, and dyslipidemia) and leads to a higher risk of cardiometabolic dysregulation, metabolic syndrome, and cardiovascular mortality. ${ }^{17,34,36,37}$ High levels of physical fitness attenuate the association between obesity and clustered cardiometabolic risk from childhood to adolescence. ${ }^{38}$ Our results are similar to those of a recent study that showed that Colombian children and adolescents with NWO had a significantly worse cardiometabolic profile and lower level of physical fitness compared with that of their NW lean counterparts. ${ }^{39}$ In the present study, university students classified as having NWO had higher FM and greater cardiovascular risk, lower LM and lower levels of physical fitness. NWO was associated negatively with the fitness score and was associated positively with the MetS score. Young people with NWO should be mindful of the potential hidden health risks of excess $\mathrm{BF}$ and participate in exercise to enhance physical fitness.

Our study had two main limitations. First, the number of participants with NW or NWO was relatively small. Second, because of the cross-sectional design of our study, causality could not be established. Therefore, a large study cohort and longitudinal studies are needed to determine the relationship between body composition with cardiovascular risk and physical fitness.

\section{Conclusions}

We showed that NWO prevalence was $16.7 \%$ in males and $45.3 \%$ in females. University students with NWO were characterized by lower levels of physical fitness and LM, higher FM, and greater cardiovascular risk. These students with NWO should be mindful of the potential hidden health risks of excess $\mathrm{BF}$ and participate in exercise to enhance physical fitness.

\section{Practical Applications}

Individuals with NWO tend to develop certain characteristic health conditions, such as excess BF and lower levels of physical fitness, which lead to a high risk of cardiovascular disease. Screening for excess BF in individuals with normal BMI could better identify those at a higher risk for cardiometabolic disturbances. Individuals with NWO should be made aware of the potential hidden health risks of excess $\mathrm{BF}$ and poor physical fitness. Proactive efforts must be made to encourage exercise to lose weight and enhance physical fitness.

\section{Data Sharing Statement}

Data are available from the corresponding author upon reasonable request.

\section{Acknowledgments}

This study was undertaken at the Shanghai University of Sport in Shanghai, China. The authors thank all the staff members from School of Kinesiology, Shanghai University of Sport, for their help in data collection. We also thank the participants for their contributions to this study.

\section{Author Contributions}

All authors made a significant contribution to the work reported, whether in the conception, study design, execution, acquisition, analysis and interpretation of data, or in all these areas. They took part in the drafting, revising or critical reviewing of the manuscript; gave final approval of the version to be published, agreed on the journal to which the article should be been submitted, and agreed to be accountable for all aspects of the work.

\section{Funding}

This study was funded by the National Natural Science Foundation of China $(31671242,31971097)$ and the Construction Project of High-Level Local Universities in Shanghai, China.

\section{Disclosure}

The authors report no conflicts of interest in this work.

\section{References}

1. Swinburn BA, Kraak VI, Allender S, et al. The global syndemic of obesity, undernutrition, and climate change: the Lancet Commission Report. Lancet. 2019;393(10173):791-846. doi:10.1016/S01406736(18)32822-8

2. Zheng Y, Manson JE, Yuan C, et al. Associations of weight gain from early to middle adulthood with major health outcomes later in life. JAMA. 2017;318(3):255-269. doi:10.1001/jama.2017.7092

3. Jia G, Shu XO, Liu Y, et al. Association of adult weight gain with major health outcomes among middle-aged Chinese persons with low body weight in early adulthood. JAMA Netw Open. 2019;2(12): e1917371. doi:10.1001/jamanetworkopen.2019.17371

4. Adab P, Pallan M, Whincup PH. Is BMI the best measure of obesity? BMJ. 2018;360:k1274. doi:10.1136/bmj.k1274

5. Bazzocchi A, Ponti F, Albisinni U, Battista G, Guglielmi G. DXA: technical aspects and application. Eur $J$ Radiol. 2016;85 (8):1481-1492. doi:10.1016/j.ejrad.2016.04.004

6. Poirier P, Giles TD, Bray GA, et al. Obesity and cardiovascular disease: pathophysiology, evaluation, and effect of weight loss: an update of the 1997 American Heart Association Scientific Statement on Obesity and Heart Disease from the Obesity Committee of the Council on Nutrition, Physical Activity, and Metabolism. Circulation. 2006;113(6):898-918. 
7. Oliveros E, Somers VK, Sochor O, Goel K, Lopez-Jimenez F. The concept of normal weight obesity. Prog Cardiovasc Dis. 2014;56 (4):426-433. doi:10.1016/j.pcad.2013.10.003

8. Mattila VM, Tallroth K, Marttinen M, Pihlajamaki H. Physical fitness and performance. Body composition by DEXA and its association with physical fitness in 140 conscripts. Med Sci Sports Exerc. 2007;39(12):2242-2247. doi:10.1249/mss.0b013e318155a813

9. Liao Y, Chang SH, Miyashita M, et al. Associations between health-related physical fitness and obesity in Taiwanese youth. $J \quad$ Sports Sci. 2013;31(16):1797-1804. doi:10.1080/ 02640414.2013.803588

10. Henriksson P, Cadenas-Sanchez C, Leppanen MH, et al. Associations of fat mass and fat-free mass with physical fitness in 4-year-old children: results from the MINISTOP Trial. Nutrients. 2016;8 (8):473. doi:10.3390/nu8080473

11. Kim TN, Park MS, Kim YJ, et al. Association of low muscle mass and combined low muscle mass and visceral obesity with low cardiorespiratory fitness. PLoS One. 2014;9(6):e100118. doi:10.1371/journal.pone. 0100118

12. Sato M, Kodama S, Sugawara A, Saito K, Sone H. Physical fitness during adolescence and adult mortality. Epidemiology. 2009;20 (3):463-464. doi:10.1097/EDE.0b013e31819ed09f

13. Romero-Corral A, Somers VK, Sierra-Johnson J, et al. Normal weight obesity: a risk factor for cardiometabolic dysregulation and cardiovascular mortality. Eur Heart J. 2010;31(6):737-746. doi:10.1093/eurheartj/ehp487

14. Song Y, Lei YT, Hu PJ, Zhang B, Ma J. [Situation analysis of physical fitness among Chinese Han students in 2014]. Beijing Da Xие Xиe Bao Yi Xue Ban. 2018;50(3):436-442. Chinese.

15. Mannisto S, Harald K, Kontto J, et al. Dietary and lifestyle characteristics associated with normal-weight obesity: the National FINRISK 2007 Study. $B r \quad J \quad$ Nutr. 2014;111(5):887-894. doi: $10.1017 / \mathrm{S} 0007114513002742$

16. Pickering TG, Hall JE, Appel LJ, et al. Recommendations for blood pressure measurement in humans and experimental animals: part 1: blood pressure measurement in humans: a statement for professionals from the Subcommittee of Professional and Public Education of the American Heart Association Council on High Blood Pressure Research. Circulation. 2005;111(5):697-716.

17. Wiklund P, Tormakangas T, Shi Y, et al. Normal-weight obesity and cardiometabolic risk: a 7-year longitudinal study in girls from prepuberty to early adulthood. Obesity. 2017;25(6):1077-1082. doi:10.1002/oby.21838

18. Marques-Vidal P, Pecoud A, Hayoz D, et al. Prevalence of normal weight obesity in Switzerland: effect of various definitions. Eur J Nutr. 2008;47(5):251-257. doi:10.1007/s00394-008-0719-6

19. Jia A, Xu S, Xing Y, et al. Prevalence and cardiometabolic risks of normal weight obesity in Chinese population: a nationwide study. Nutr Metab Cardiovasc Dis. 2018;28(10):1045-1053. doi:10.1016/j. numecd.2018.06.015

20. Santana CCA, Azevedo LB, Cattuzzo MT, Hill JO, Andrade LP, Prado WL. Physical fitness and academic performance in youth: a systematic review. Scand J Med Sci Sports. 2017;27(6):579-603. doi: $10.1111 /$ sms. 12773

21. Fogelholm M, Stigman S, Huisman T, Metsamuuronen J. Physical fitness in adolescents with normal weight and overweight. Scand J Med Sci Sports. 2008;18(2):162-170. doi:10.1111/j.1600-0838.2007.00685.x

22. Dong Y, Lau PWC, Dong B, et al. Trends in physical fitness, growth, and nutritional status of Chinese children and adolescents: a retrospective analysis of 1.5 million students from six successive national surveys between 1985 and 2014. Lancet Child Adolesc Health. 2019;3(12):871-880. doi:10.1016/S2352-4642(19)30302-5
23. Foulis SA, Hughes JM, Friedl KE. New concerns about military recruits with metabolic obesity but normal weight ("skinny fat"). Obesity. 2020;28(2):223. doi:10.1002/oby.22724

24. Finelli C, Sommella L, Gioia S, La Sala N, Tarantino G. Should visceral fat be reduced to increase longevity? Ageing Res Rev. 2013;12(4):996-1004. doi:10.1016/j.arr.2013.05.007

25. Menni C, Migaud M, Glastonbury CA, et al. Metabolomic profiling to dissect the role of visceral fat in cardiometabolic health. Obesity. 2016;24(6):1380-1388. doi:10.1002/oby.21488

26. Wolfe RR. The underappreciated role of muscle in health and disease. Am J Clin Nutr. 2006;84(3):475-482.

27. Artero EG, Ruiz JR, Ortega FB, et al. Muscular and cardiorespiratory fitness are independently associated with metabolic risk in adolescents: the HELENA study. Pediatr Diabetes. 2011;12(8):704-712. doi:10.1111/j.1399-5448.2011.00769.x

28. Litwin SE. Normal weight obesity: is bigger really badder? Circ Cardiovasc Imaging. 2012;5(3):286-288. doi:10.1161/ CIRCIMAGING.112.974840

29. Zhang M, Schumann M, Huang T, Tormakangas T, Cheng S. Normal weight obesity and physical fitness in Chinese university students: an overlooked association. BMC Public Health. 2018;18(1):1334. doi:10.1186/s12889-018-6238-3

30. Guo S, Huang Y, Zhang Y, Huang H, Hong S, Liu T. Impacts of exercise interventions on different diseases and organ functions in mice. $J$ Sport Health Sci. 2020;9(1):53-73. doi:10.1016/j. jshs.2019.07.004

31. Luan $\mathrm{X}$, Tian $\mathrm{X}$, Zhang $\mathrm{H}$, et al. Exercise as a prescription for patients with various diseases. J Sport Health Sci. 2019;8 (5):422-441. doi:10.1016/j.jshs.2019.04.002

32. Wang R, Tian H, Guo D, Tian Q, Yao T, Kong X. Impacts of exercise intervention on various diseases in rats. J Sport Health Sci. 2020;9 (3):211-227. doi:10.1016/j.jshs.2019.09.008

33. WHO. Recommended levels of physical activity for adults aged 18-64 years. Available from: https://www.who.int/dietphysicalactiv ity/factsheet_adults/en/. Accessed May 28, 2020.

34. Marques-Vidal P, Pecoud A, Hayoz D, et al. Normal weight obesity: relationship with lipids, glycaemic status, liver enzymes and inflammation. Nutr Metab Cardiovasc Dis. 2010;20(9):669-675. doi:10.1016/j.numecd.2009.06.001

35. Correa-Rodriguez M, Gonzalez-Ruiz K, Rincon-Pabon D, et al. Normal-weight obesity is associated with increased cardiometabolic risk in young adults. Nutrients. 2020;12(4):1106. doi:10.3390/ nu12041106

36. De Lorenzo A, Martinoli R, Vaia F, Di Renzo L. Normal weight obese (NWO) women: an evaluation of a candidate new syndrome. Nutr Metab Cardiovasc Dis. 2006;16(8):513-523. doi:10.1016/j. numecd.2005.10.010

37. Jean N, Somers VK, Sochor O, Medina-Inojosa J, Llano EM, LopezJimenez F. Normal-weight obesity: implications for cardiovascular health. Curr Atheroscler Rep. 2014;16(12):464. doi:10.1007/s11883014-0464-7

38. Brouwer SI, Stolk RP, Liem ET, Lemmink KA, Corpeleijn E. The role of fitness in the association between fatness and cardiometabolic risk from childhood to adolescence. Pediatr Diabetes. 2013;14 (1):57-65. doi:10.1111/j.1399-5448.2012.00893.x

39. Garcia-Hermoso A, Agostinis-Sobrinho C, Camargo-Villalba GE, et al. Normal-weight obesity is associated with poorer cardiometabolic profile and lower physical fitness levels in children and adolescents. Nutrients. 2020;12(4):1171. doi:10.3390/ nu12041171 


\section{Publish your work in this journal}

Diabetes, Metabolic Syndrome and Obesity: Targets and Therapy is an international, peer-reviewed open-access journal committed to the rapid publication of the latest laboratory and clinical findings in the fields of diabetes, metabolic syndrome and obesity research. Original research, review, case reports, hypothesis formation, expert opinion and commentaries are all considered for publication. The manuscript management system is completely online and includes a very quick and fair peer-review system, which is all easy to use. Visit http://www.dovepress.com/testimonials.php to read real quotes from published authors.

Submit your manuscript here: https://www.dovepress.com/diabetes-metabolic-syndrome-and-obesity-targets-and-therapy-journal 